\title{
The Spallation Neutron Source Project - Physical and Technical Challenges ${ }^{1}$
}

\author{
Jie Wei ${ }^{2}$ \\ for the Spallation Neutron Source Collaboration, USA
}

Abstract. The Spallation Neutron Source (SNS) is designed to reach an average proton beam power of $1.4 \mathrm{MW}$ for pulsed neutron production. This paper summarizes design aspects and physical challenges to the project.

\section{CONTENTS}

Introduction

Design Philosophy

Accelerator Design Choices

Superconducting vs. Warm Linac . . . . . . . 3

Accumulator Ring vs. RCS . . . . . . . . . . 3

Ring's FODO-doublet Lattice . . . . . . . . . 3

Challenges \& Lessons Learned

Front End \& Warm Linac .

Superconducting RF Linac $\ldots \ldots \ldots \ldots$

Ring and Transport ........... 5

Summary

\section{INTRODUCTION}

The SNS project, designed to reach an average beam power above 1.4 MW for pulsed neutron production, is presently in the fourth year of a seven-year construction cycle at ORNL (Fig. 1) [1]. The accelerator system operates at a repetition rate of $60 \mathrm{~Hz}$ and an average current of $1.6 \mathrm{~mA}$. It consists of an $\mathrm{H}^{-} \mathrm{RF}$ volume source of $48 \mathrm{~mA}$ peak current at $6 \%$ duty, a low-energy beam transpoit (LEBT) housing a first-stage beam chopper with $\pm 20 \mathrm{~ns}$ rise/fall time; a $402.5 \mathrm{MHz}$, 4-vane radio-frequencyquadrupole (RFQ); a medium-energy beam transport (MEBT) housing a second-stage chopper $(< \pm 10 \mathrm{~ns}$ rise/fall), an adjustable beam-halo scraper, diagnostics devices, and matching quadrupoles; a $402.5 \mathrm{MHz}$, 6-tank drift-tube-linac (DTL) with permanent-magnet quadrupoles; a $805 \mathrm{MHz}$, 4-module coupled-cavity-linac (CCL); a $805 \mathrm{MHz}$, superconducting RF (SRF) linac of

${ }^{1}$ SNS is managed by UT-Battelle, LLC, under contract DE-AC05OOOR22725 for the U.S. Department of Energy. SNS is a partnership of six national laboratories: Argonne, Brookhaven, Jefferson, Lawrence Berkeley, Los Alamos, and Oak Ridge.

2 Brookhaven National Laboratory, and on a joint appointment with the Oak Ridge National Laboratory for the SNS Project.

C 2001 AIP The Spallation Neutron Source Project -

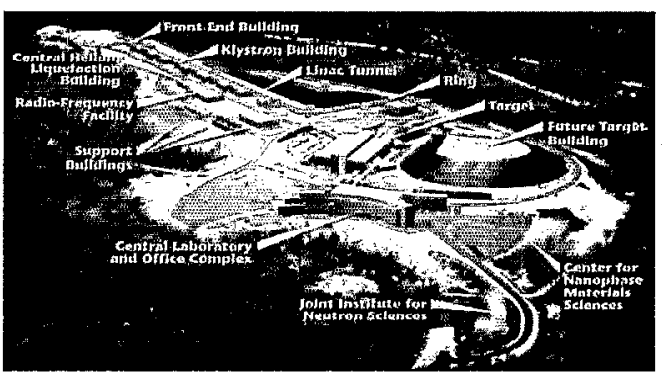

FIGURE 1. Layout of the Spallation Neutron Source.

TABLE 1. Spallation Neutron Source primary parameters.

\begin{tabular}{lrr}
\hline & Baseline & Back-up \\
\hline Kinetic energy, $E_{k}[\mathrm{MeV}]$ & 1000 & 975 \\
Uncertainty, $\Delta E_{k}(95 \%)[\mathrm{MeV}]$ & \pm 15 & \pm 15 \\
SRF cryo-module number & $11+12$ & $11+15$ \\
SRF cavity number & $33+48$ & $33+60$ \\
Peak field $E_{p}(\beta=0.61)[\mathrm{MV} / \mathrm{m}]$ & 27.5 & 27.5 \\
$\Delta E_{p}(\beta=0.61)[\mathrm{MV} / \mathrm{m}]$ & \pm 2.5 & \pm 2.5 \\
Peak field $E_{p}(\beta=0.81)[\mathrm{MV} / \mathrm{m}]$ & 35 & 27.5 \\
$\Delta E_{p}(\beta=0.81)[\mathrm{MV} / \mathrm{m}]$ & $+2.5 /-7.5$ & \pm 2.5 \\
Beam power on target, $P_{\max }[\mathrm{MW}]$ & 1.4 & 1.7 \\
Pulse length on target [ns] & 695 & 699 \\
Chopper beam-on duty factor [\%] & 68 & 68 \\
Linac macro pulse duty factor [\%] & 6.0 & 6.0 \\
Ave. macropulse $\mathrm{H}^{-}$current $[\mathrm{mA}]$ & 26 & 32 \\
Linac ave. beam current $[\mathrm{mA}]$ & 1.6 & 1.9 \\
Ring rf frequency $[\mathrm{MHz}]$ & 1.058 & 1.054 \\
Ring injection time $[\mathrm{ms}]$ & 1.0 & 1.0 \\
Ring bunch intensity $\left[10^{14}\right]$ & 1.6 & 1.9 \\
Ring spacc-chargc tunc sprcad & 0.15 & 0.20 \\
\hline
\end{tabular}

medium- and high- $\beta$ cavities accelerating the beam to the full energy; a high-energy beam transport (HEBT) for diagnostics, transverse and longitudinal collimation, matching, energy correction and painting; and an accumulator ring compressing the $1 \mathrm{GeV}, 1 \mathrm{~ms}$ pulse to $650 \mathrm{~ns}$ for delivery onto the target through a ring-target beam transport (RTBT).

Table 1 lists major parameters. The energy acceptance of the ring is about $\pm 50 \mathrm{MeV}$, mainly due to conditions

Physical and Technical Challenges ${ }^{3} \quad 2002 / 5 / 30$ 


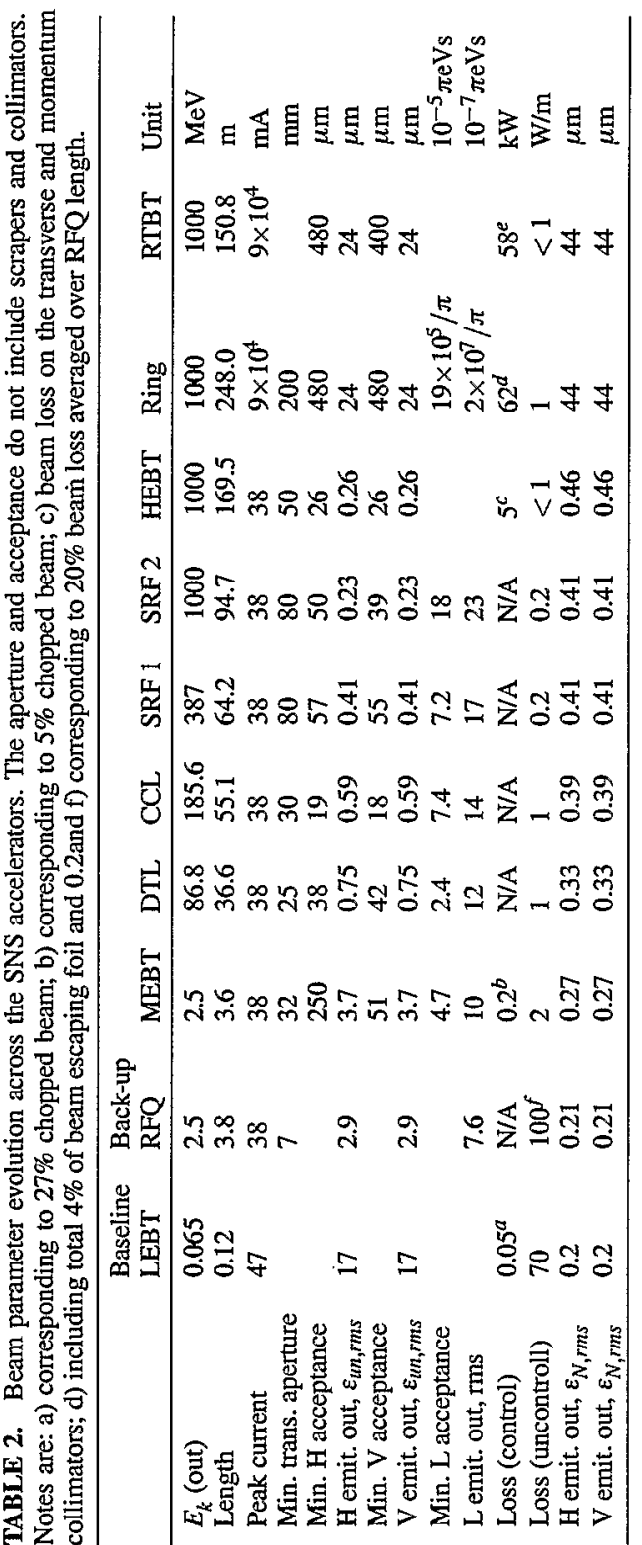

for a tolcrable $\mathrm{H}^{-}$and $\mathrm{H}^{0}$ stripping loss. The back-up scenario corresponds to the case if the surface field of the SRF cavity is lower than expected ( $37.5 \mathrm{MV} / \mathrm{m})$. Extra tunnel space $(71 \mathrm{~m})$ is reserved to extend the linac length for a higher output energy. Table 2 shows evolulion of beam parameters during the cycle including expected energy, horizontal $(\mathrm{H})$, vertical $(\mathrm{V})$, and longitudinal (L) acceptances and emittances, and controlled and uncontrolled beam losses.

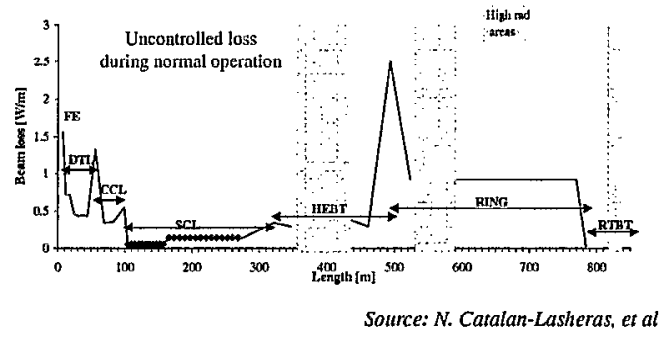

FIGURE 2. Expected beam loss across the SNS accelerator complex. The uncontrolled beam loss is at $1 \mathrm{~W} / \mathrm{m}$ level.

\section{DESIGN PHILOSOPHY}

The primary concern is that radio-activation caused by excessive uncontrolled beam loss can limit the machine's availability and maintainability. Based on operational experiences, hands-on maintenance demands that the average uncontrolled heam loss does not exceed $1 \mathrm{~W}$ beam power per tunnel-meter [2]. Uncontrolled losses are usually attributed to 1) mismatch upon change of linac structure, lattice, and frequency; 2) space-charge effects including envelope and parametric resonances and nonequipartition in the linac, and resonance crossing and instability enhancement in ring; 3) limited physical and momentum acceptance; 4) premature $\mathrm{H}^{-}$and $\mathrm{H}^{0}$ stripping and ring injection foil scattering; 5) magnetic errors, fringe fields, and misalignments; 6) instabilities (resistive impedances due to e.g. extraction kicker, and electron cloud); and 7) accidental loss due to system malfunction (ion source and linac, ring extraction kickers).

SNS addresses the above seven issues by adopting a low-loss design philosophy [3]. Above all, foreseen losses are localized to shielded areas using 1) adjustablc scrapers in the MEBT; 2) transverse and momentum collimators in the HEBT prior ring injection; 3) twostage transverse collimation and momentum cleaning with beam-in-gap (BIG) kicker in the ring; 4 ) collimator protection in the RTBT, and 5) beam-gap cleaning with LEBT and MEBT choppers and ring BIG kicker (Fig. 2).

Emphasis is also put on machine's flexibility and reliability. The SRF linac allows operation with one failed cavity/klystron; the ring accepts $\pm 5 \%$ variation in linac output energy; a wide ring tuning range avoiđs resonances; a robust injection allows independent horizontal, vertical, and longitudinal painting; adjustable collimation systems accommodate variable beam size; and design reserve and redundancy ensure a high availability (e.g., spare cryo-module for a quick replacement, power supplies compatible with $1.3 \mathrm{GeV}$ energy, multi-foil exchange, spare kicker power supply (PFN), and aperture clearance for one-kicker failure).

Finally, the facility is designed with the potential to reach a beam energy up to $1.3 \mathrm{GeV}$ and a beam power higher than $2 \mathrm{MW}$, capable of supplying a second neu-

Physical and Technical Challenges ${ }^{4} \quad 2002 / 5 / 30$ 


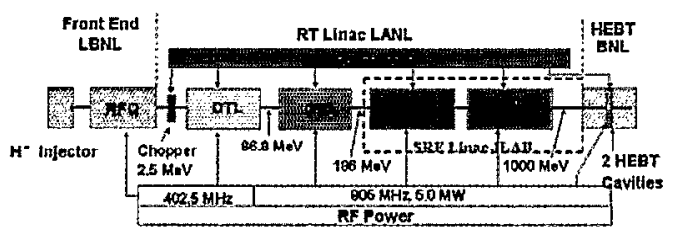

FIGURE 3. SNS front-end and linac stucture.

tron target. The higher energy can be reached by upgrading the superconducting RF cavity gradient and klystron power supplies, and by filling the presently unoccupied linac tunnel spaces with up to 9 additional cryo-modules. The ring is capable of accommodating the energy and power increase without extensive hardware change space is reserved for two additional extraction kickers, and for the replacement of 2 injection-chicane dipoles to satisfy $\mathrm{H}^{0}$ stripping conditions [4].

\section{ACCELERATOR DESIGN CHOICES}

\section{Superconducting vs. Warm Linac}

The SRF linac operating at $805 \mathrm{MHz}$ frequency accelerates the $\mathrm{II}^{-}$beam from $186 \mathrm{MeV}$ to top energy (Fig. 3). Comparing with the original normal-conducting (warm) CCL linac, the SRF linac provides a high accelerating gradient $(11-16 \mathrm{MV} / \mathrm{m})$ capable of reaching a higher beam energy, encounters less beam loss and halo scraping due to its larger bore radius, is immune to one cavity/klystron failure, operates at a better vacuum, and is expected to have higher reliability and availability. The selection of two types of SRF cavities allows for economic savings and future energy upgrades. On the other hand, the relatively large phase slip requires detailed error-sensitivity analysis. The choice of cavity geometric $\beta$ value is based on a smooth transition from the warm section linac, a maximized final output energy, and a comfortable transition from medium- to high- $\beta$ section with tolerance to one cavity failure. We also choose constant-gradient, continuous focusing to maximize the accelerating field strength [5].

Considering the tight construction schedule, a moderate peak surface field of $27.5 \mathrm{MV} / \mathrm{m}$ is chosen for the medium- $\beta$ cavity. Benefiting from electro-polishing, a higher peak field of $35 \mathrm{MV} / \mathrm{m}$ is assumed for the high$\beta$ cavity. In order to reduce uncertainties in RF controls of an ion $(\beta<1)$ beam under Lorentz detuning, microphonics, beam transients and injecting energy offset, we decide to drive each cavity with its own klystron using independent amplitude and phase control.

\section{Accumulator Ring vs. RCS}

During the first year of construction, a study was performed comparing the present structure of full-

(C) 2001 AIP The Spallation Neutron Source Project -

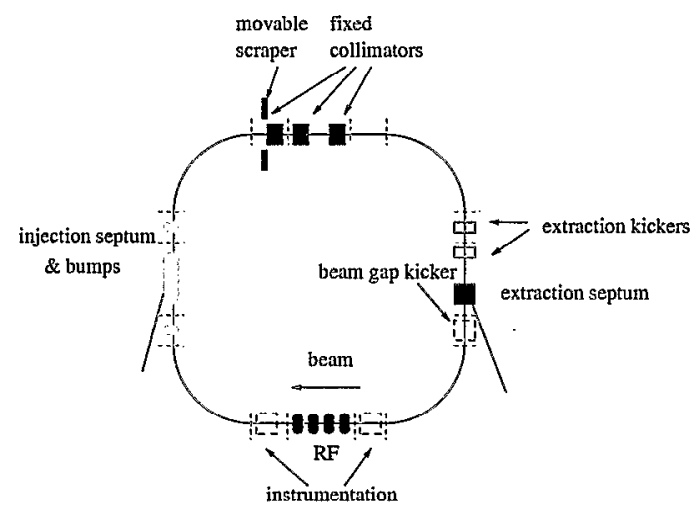

FIGURE 4. SNS accumulator ring layout.

energy linac plus accumulator ring to a rapid-cyclingsynchrotron (RCS) design: a $60 \mathrm{~Hz}, 400 \mathrm{MeV}$ linac feeds two, vertically stacked RCSs accelerating the proton beam to $2 \mathrm{GeV}$ energy. The biggest challenge to the RCS design is from the stringent $(1 \mathrm{~W} / \mathrm{m})$ beam-loss criterion: although relaxed by a factor of 5 , still only $0.4 \%$ uncontrolled loss is allowed for a 2 MW beam power assuming $90 \%$ collimation efficiency. On the other hand, among existing rings the lowest loss of about $0.3 \%$ is achieved al LANL's PSR, a $800 \mathrm{MeV}$ accunulator, as opposed to typical losses of a few to tens of percent in RCSs (e.g. ISIS, FNAL and AGS Boosters).

As opposed to the accumulator, the RCSs operating at $30 \mathrm{~Hz}$ require a high $\mathrm{RF}$ voltage (about $400 \mathrm{kV}$ per ring at $1.4-1.9 \mathrm{MHz}$ ) for fast acceleration, a large magnet aperture to accommodate the space charge at a lower energy, ceramic vacuum pipes with detailed RF shielding, and high-performance power supplies. Minimization of magnetic errors due to eddy current, ramping, saturation, and power-supply tracking is non-trivial. 'l'he study concluded that the required RCS design is technically more demanding and less cost effective [4].

Permanent magnets were considered as an option for the accumulator ring magnets. Electromagnetic magnets were chosen instead, given the uncertainty in the linac energy. This choice is especially appropriate to accommodate later-adopted SRF linac.

\section{Ring's FODO-doublet Lattice}

The four-fold symmetric ring lattice contains four dispersion-free straights, each housing injection, collimation, RF, and extraction, as shown in Fig. 4. Each achromatic arc consists of 4 FODO cells with $90^{\circ}$ horizontal phase advance.

After optimization, the ring lattice has doublet straights [3]. The lattice combines the FODO structure's simplicity and ease of correction with the doublet structure's flexibility for injection and collimation. In- 


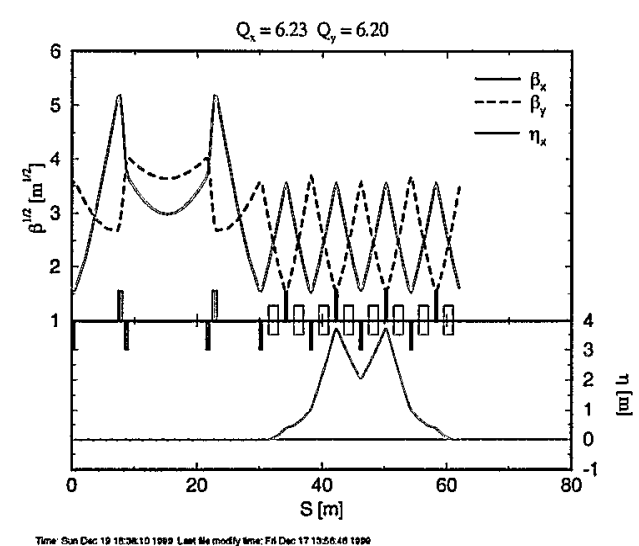

FIGURE 5. SNS ring lattice super-period of FODO/doublet structure. The lattice periodicity is 4 .

jection at a dispersion-free region allows independently adjustable painting in the transverse (with orbit bumps in the ring) and longitudinal (with an energy-spreading phase-modulated RF cavity in the HEBT) directions for a robust operation. The $12.5 \mathrm{~m}$-long uninterrupted straight section with a flexible phase advance further improves collimation efficiency. Comparing with the original all-FODO lattice, matching between the arcs and the straights increases the arc acceptance by $50 \%$ with the same magnet aperture (Fig. 5).

\section{CHALLENGES \& LESSONS LEARNED \\ Front End \& Warm Linac}

Tight optical focusing used for chopping and antichopping in a long MEBT is a source of beam-halo generation. Studies show that even without the antichopper, partially deflected particles are still mostly contained by the envelope of the nominal unchopped beam [6]. The MEBT quadrupoles are thus made independently adjustable so that alternative optics can be realized, avoiding tight focusing at the antichopper or MEBT chopper.

Permanent-magnet quadrupoles are used in the DTL due to the tight geometry $(402.5 \mathrm{MHz}$ starting at 2.5 $\mathrm{MeV}$ ), although electromagnetic quadrupoles could be used at DTL tank 3 and beyond. During 1999, the aperture of CCL was reduced from 4 to $3 \mathrm{~cm}$ for cost savings. Later when SRF linac is adopted, simulated beam loss often occurs near the end of CCL as the focusing strength is reduced to match the SRF optics.

A key challenge in linac performance is to minimize beam emittance growth and centroid jitter in both transverse and longitudinal directions upon ring injection, reducing foil traversal, scattering and radio-activation. The warm DTL operating at $402.5 \mathrm{MHz}$ is expected to be less

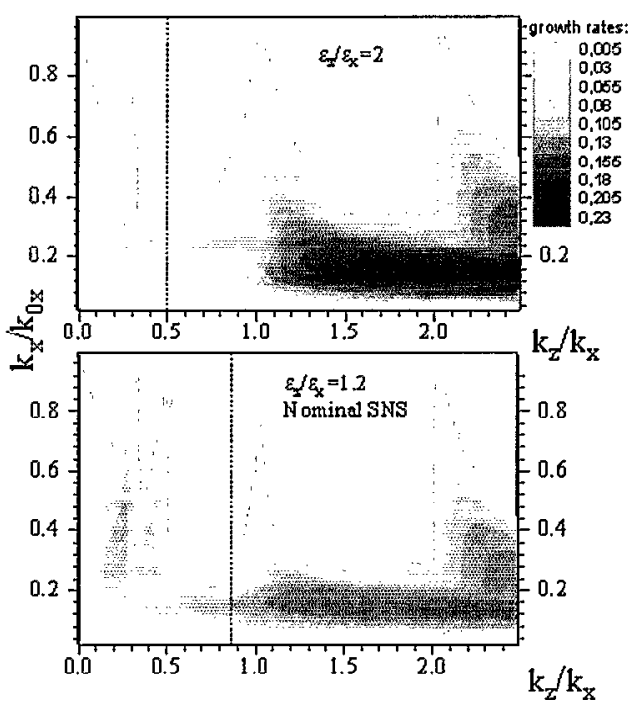

Source: I. Hofmann

FIGURE 6. Analytical resonance chart showing Instability growth rate due to space-charge coupling resonance. The effects become important only when the transverse and longitudinal tunes are on resonance, and when the emittances differ significantly. The dash lines indicate equipartition.

sensitive to vibrational noises than most existing linacs operating at $200 \mathrm{MHz}$. A tight RF control ( $<0.5 \%$ amplitude and $0.5^{\circ}$ phase error) warrants tolerable energy variation before the beam enters energy correction and spreading cavities in the HEBT [7].

\section{Supcrconducting RF Linac}

Using only two types of cavity $\beta$ for over $800 \mathrm{MeV}$ of acceleration compromises the equipartition law. Spacecharge coupling can cause transverse and longitudinal emittance exchange when the emittance ratio meets resonance conditions (Fig. 6) [8]. In addition, depending on the level of initial mismatch, space-charge parametric halo may develop in the linac. Efforts were made to reserve an economically affordable large aperture, and to reserve tunability in the MEBT, CCL and SRF linac.

Effects of higher-order modes (HOM) on the cavities is another issue. Overlapping of beam and HOM spectrum is possible because of the pulsed time structure of the beam and the fact that the beam frequency shifts with variable ring energy and repetition rate (e.g. for some two-target operation scenarios). Fortunately, transverse and longitudinal (beam break-up) instabilities are minor issues for an ion beam in the presence of a cavity-tocavity frequency spread [9]. HOM dampers are implemented only for the purpose of power dissipation [10]. 


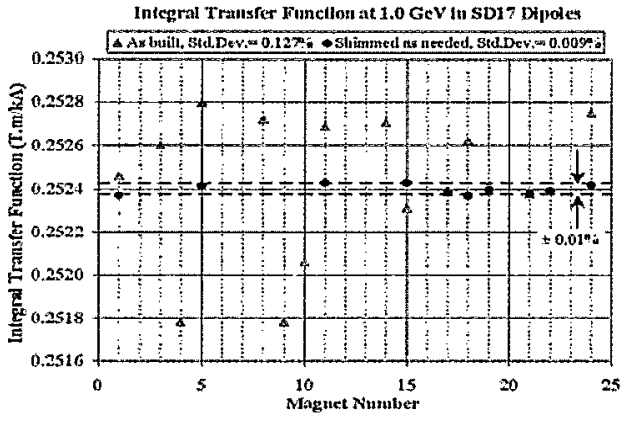

Source: P. Wanderer, A. Jain, ef al

FIGURE 7. Variation of integral transfer function of SNS ring dipole magnets before and after shimming. The measurement current corresponds to $1 \mathrm{GeV}$ beam energy.

The SRF linac performance is limited by the available klystrons power (550 kW). Up to $40 \% \mathrm{RF}$-power is reserved for compensation of cavity errors (Lorntz detuning, microphonics, coupling loss, frequency setting), klystron loss, and missing-cavity tuning. To reduce such overhead, each SRF cavity is equipped with a piezo crystal driven fast tuner to compensate for the Lorentz force.

\section{Ring and Transport}

Solid-steel, as opposed to laminated-steel, was selected for most ring and transport magnet cores for cost savings. Individually, good field quality $\left(<10^{-4}\right.$ relative error at full acceptance) is achieved. However, excessive (up to $0.25 \%$ ) magnet-to-magnet variation is found in the dipole transfer function and its current dependence, as shown in Fig. 7 [11]. These dipoles are shimmed to achieve below $10^{-4}$ variation for $1 \mathrm{GeV}$ operation, and sorted according to $1.3 \mathrm{GeV}$ measurement data to minimize orbit corrector strength.

Main ring challenges include meeting the target requirements on the peak current density, minimizing uncontrolled beam loss, and controlling collective effects (space charge, instabilities, electron cloud (Fig. 8)) [4]. Efforts are made to minimize leading sources of beamcoupling impedance (Fig. 9 [12]), and to enhance Landau damping [4].

High-performance beam diagnostics is needed to accommodate the large variation of beam parameters, and for machine protection across the entire facility. Laserwire monitors are under test for possible implementation in the SRF linac for a clean operalion, and luminescence profile monitors are under test to reduce space-charge and electron-cloud complications in the ring.

\section{SUMMARY}

By adopting superconducting RF technology for the linac and by fully optimizing the accumulator ring de-

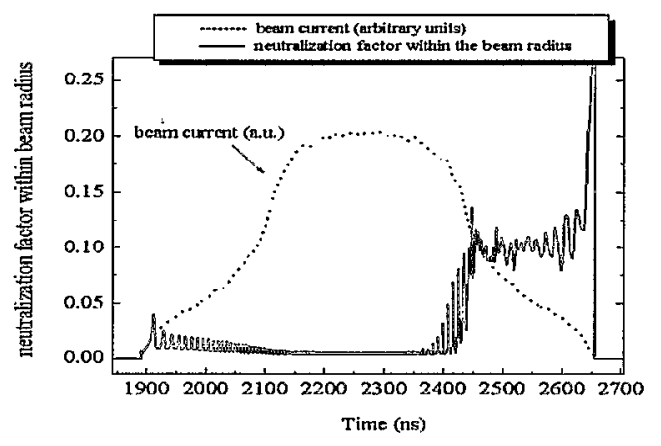

Source: M. Pivi, M. Furman

FIGURE 8. Simulation of single-bunch electron multipacting with a peak secondary-emission yield of 2 .
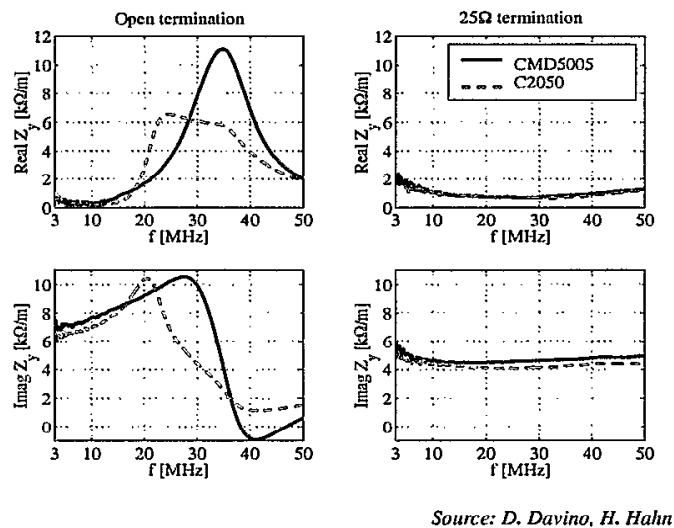

FIGURE 9. Comparison of bench-measured coupling impcdance for opcn and $25 \Omega$ PFN termination, and high (1600) and medium (100) permeability ferrite of the ring extraction-kicker assembly.

sign, the Spallation Neutron Source project, half way towards its completion, is meeting the challenge to be a next-generation, high-power accelerator facility.

I am indebted to my colleagues, especially those participating in SNS accelerator-physics discussions.

\section{REFERENCES}

1. N. Holtkamp, EPAC (2002)

2. N. Catalan-Lasheras et al, SNS Notes SNS/AP/7 (2001)

3. J. Wei et al, Phys. Rev. ST-AB 3, 080101 (2000)

4. J. Wei et al, EPAC'00, 981; PAC (2001) 319; EPAC (2002)

5. J. Stovall et al, LINAC 2000, p. 605

6. D. Jeon et al, SNS 104050000-TD0010-R01 (2002)

7. D. Raparia et al, Workshop HB2002, FNAL (2002)

8. I. Hofmann, ct al, PAC (2001) 2902

9. R. Sundelin et al PAC'01 1984; D. Jeon et al, PAC'01 2063

10. S. Kim, et al, PAC (2001) 1128

11. P. Wanderer et al, EPAC (2002)

12. D. Davino et al, EPAC (2002)

Physical and Technical Challenges $^{7} \quad$ 2002/5/30 